Arch Womens Ment Health. 2018 October ; 21(5): 583-586. doi:10.1007/s00737-018-0836-z.

\title{
An Open-Label Pilot Study of a Home Wearable Light Therapy Device for Postpartum Depression
}

\author{
Leslie M. Swanson ${ }^{a}$, Helen J. Burgess ${ }^{b}$, Jennifer Zollars ${ }^{a}$, and J. Todd Arnedt ${ }^{a}$ \\ aDepartment of Psychiatry, University of Michigan, Ann Arbor, Michigan, United States \\ ${ }^{\mathrm{b}}$ Rush University Medical Center, Chicago, Illinois, United States
}

\begin{abstract}
We sought to establish the feasibility and preliminary effects of home wearable light therapy for postpartum depression, and its effects on circadian measures. Eight women within 6 months postpartum were prescribed 60 minutes of daily morning light therapy for 5 weeks. The device was well-tolerated. Significant improvements were observed in self-report and clinician-rated depression symptoms, with little change in objective circadian measures. Home wearable light therapy is feasible for postpartum women and may be a promising treatment for postpartum depression.
\end{abstract}

\section{Keywords}

Depression; postpartum; light therapy; circadian

\section{INTRODUCTION}

As many as $20 \%$ of postpartum women in the United States experience symptoms of depression (Gavin et al. 2005), and many perinatal women prefer non-pharmacological treatments (O'Mahen and Flynn 2008). Morning light therapy holds promise for postpartum depression by targeting sleep and circadian rhythms, both of which play a role in postpartum depression. Sleep disturbance is an established risk factor for postpartum depression (Okun 2016), and light therapy is effective for sleep problems (van Maanen et al. 2016). Emerging work also implicates later timing of circadian rhythms in postpartum depression, as demonstrated by a phase delay of melatonin onset (Sharkey et al. 2013) and higher morning melatonin levels (Parry et al. 2008). Morning light therapy typically corrects phase delays by advancing the circadian timing system (Lewy et al. 1998).

To date, only two small trials have evaluated morning light therapy for postpartum depression: an open pilot, which showed improvements in depression scores after 4 weeks (Corral et al. 2000); and a small randomized controlled trial, which showed no difference from placebo (Corral et al. 2007). These studies used a standard light box, which may have

*Corresponding Author: Leslie M. Swanson, 4250 Plymouth Rd, Ann Arbor, MI 48109, LMSwan@med.umich.edu, Phone: 734-764-1234 Fax: 734-764-1229.

Conflict of Interest: Authors Swanson, Burgess, Zollars, and Arnedt declare that they have no conflict of interest. 
influenced adherence. New wearable devices deliver light therapy in portable glasses, which may be easier for postpartum women to use. However, these devices have not been systematically evaluated for perinatal depression nor have studies examined the effects of light therapy on circadian measures in postpartum women. In the present study, we sought to establish the feasibility of a commercially-available home wearable light therapy device (ReTimer®; Adelaide, AU, http://re-timer.com) in postpartum women with depression, assess its preliminary effects on depression, examine sleep and circadian changes, and evaluate relationships between circadian measures and mood using a novel, home-based circadian assessment protocol.

\section{METHODS}

Potential participants were recruited from the Department of Psychiatry at the University of Michigan and the greater Ann Arbor community. Participants were eligible if they were $\geq 18$ years of age, $\leq 6$ months postpartum, met DSM-V criteria for major depressive disorder, and scored $\geq 20$ on the Structured Interview Guide for the Hamilton Depression Rating ScaleSeasonal Affective Disorder (SIGH-SAD). Major exclusion criteria included other psychiatric conditions (except generalized anxiety disorder), history of seizures, and use of light-sensitizing medications. Participants taking an antidepressant medication were included if their medication use was stable. Potential participants were screened for eligibility in an in-person interview. Informed consent was obtained from all individual participants included in the study. Study procedures were approved by the University of Michigan Medical School Institutional Review Board. This study was registered on clinicaltrials.gov (identifier: NCT02769858).

Following a one-week pre-treatment period, all participants were provided with Re-Timer® light therapy glasses and were instructed to use them daily for 60 minutes within 30 minutes of waking for the next 5 weeks. The Re-Timer emits green-blue $500 \mathrm{~nm}$ dominant wavelength light (506 lux $\mathrm{lm} / \mathrm{m}^{2}$ at the eye); devices were purchased from the manufacturer. Wake times were not fixed. Participants recorded time and duration of use on a daily log.

Depression measures included the SIGH-SAD (minus the sleep items) completed pre- and post-treatment, and the Edinburgh Postnatal Depression Scale (EPDS; minus the sleep items) completed weekly. Side effects were assessed weekly using the Systematic Assessment for Treatment Emergent Events (SAFTEE).

Sleep assessments completed at pre- and post-treatment included one week of wrist actigraphy (Actiwatch-2, Philips Respironics; Andover, MA) and daily sleep diaries. When estimating time to fall asleep or time awake during the night, participants were instructed to exclude time spent caring for their children on the diary. Actigraphy was scored using Actiware ${ }^{\circledR}$ software (Version 6.0) in conjunction with sleep/wake diaries. Sleep outcomes included total sleep time (calculated as (time in bed - (sleep onset latency + time spent awake during the night)) and sleep efficiency (calculated as (time in bed/total sleep time)*100) per sleep diary and wrist actigraphy. 
Home-based salivary dim light melatonin onset (DLMO) was collected pre- and posttreatment, following validated procedures by Burgess (Burgess et al. 2015). Saliva was collected every 30 minutes starting $6 \mathrm{~h}$ before habitual bedtime (13 samples in total). Compliance with dim light during DLMO was monitored using a light-sensing actigraph (Actiwatch, Philips Respironics), worn around the neck to measure light exposure close to the face. A MEMS TrackCap® (Sion, $\mathrm{CH}$ ) monitored when the bottle containing swabs was opened to determine compliance with the time of sample. Ninety-eight percent of the samples were considered compliant with dim light (light <50 lux within 30 minutes before sample) and sample timing (sample within 5 minutes of scheduled time) and therefore valid for analysis.

Circadian measures included time of DLMO and phase angle difference (PAD). Time of DLMO was determined using linear interpolation of the clock time at which the melatonin level exceeded the mean value of three consecutive low daytime values plus two times the standard deviations of these points. To determine PAD, we calculated the difference in time between DLMO and the midpoint of sleep per actigraphy (per a 7-day average of objective sleep onset and wake time). Shift in DLMO and PAD were calculated by subtracting pretreatment time from post-treatment time.

Paired-sample t-tests evaluated pre- to post-treatment differences in SIGH-SAD, EPDS, total sleep time per sleep diary and actigraphy, sleep efficiency per sleep diary and actigraphy, DLMO, and PAD. Effect sizes (Hedges' g) were also calculated for the mood and sleep outcomes. Correlations determined associations between the circadian and mood variables and change in these variables.

\section{RESULTS}

Ten participants were enrolled in the study. One participant dropped out prior to beginning light therapy, and a second discontinued the study after 3 weeks due to side effects (irritability and headache). Thus, our retention rate was $89 \%$ for participants who began light therapy. One participant did not have enough detectable melatonin to estimate DLMO. Phase angle difference at pre-treatment was not calculated for one participant due to malfunction of her actigraph. Most participants (75\%) completed the study in the fall-winter.

Descriptive statistics for enrolled participants are shown in Table 1. Three were using an antidepressant medication during the study (fluoxetine or bupropion). The most commonly reported side effects included headaches (56\%), irritability (44\%), and eyestrain (22\%). Participants reported using the glasses for at least 30 minutes on an average of $93 \%$ of treatment days; average start time was $28 \mathrm{~m} \pm 15.72 \mathrm{~m}$ after rise time. The average reported clock time for starting light therapy was 8:21 a.m. $\pm 1 \mathrm{~h} 48 \mathrm{~m}$.

As shown in Table 2, significant post-treatment improvements were observed in the EPDS, SIGH-SAD, and sleep efficiency per sleep diary. However, changes in total sleep time per sleep diary and actigraphy, sleep efficiency per actigraphy, DLMO, and PAD were not significant. The only significant circadian-mood correlation was found between change in PAD and percent change in SIGH-SAD score $(r=0.811, p=0.027)$, such that a lengthening 
of the PAD was associated with greater improvement on the HAMD. Given this finding, we explored DLMO changes by PAD shift type. Dim light melatonin advanced by 22 minutes in participants whose PAD lengthened $(n=2)$ vs. a 1-minute delay in those whose PAD shortened $(n=5)$, suggesting DLMO advance is in part responsible for the lengthening of the PAD.

\section{DISCUSSION}

The results of this open label pilot trial of a wearable home light therapy device suggest that the device is feasible for postpartum depression. We also found preliminary evidence to support larger-scale studies for postpartum depression. The device was well tolerated; only 1 subject discontinued participation due to side effects, and participants reported high adherence rates to the treatment protocol. As a reference, the effect sizes observed in this study for depression outcomes (0.78-1.89) are greater than the reported effect size of 0.65 from a meta-analysis of treatments (psychotherapy and medication) for perinatal depression (Sockol et al. 2011).

To our knowledge, this study is also the first to explore relationships between circadian measures and response to light therapy for postpartum depression. We found that circadian parameters did not change significantly. Without a control group, it is difficult to determine why; however, possible explanations include: 1. participants were not as adherent to therapy as they reported; 2. the timing was not optimal to produce significant advances in DLMO; 3 . our sample size was too small to detect changes. The recent discovery that light can directly impact mood without altering the circadian system by acting on intrinsically photosensitive retinal ganglion cells could explain why we observed mood improvements despite little circadian change (LeGates et al. 2014).

Our results hint that a lengthening of the PAD from pre-treatment (suggestive of an advance of circadian timing relative to sleep) may be associated with response to light therapy in this population. This is consistent with the finding that a phase delay is associated with postpartum depression (Sharkey et al. 2013), as morning light therapy should correct a delay by advancing circadian timing.

Limitations of the present study include the small sample size, uncontrolled study design, limited seasonal timing (most participants completed the study in the fall-winter), subjective report of adherence, and the use of antidepressant medications by several participants. Overall, the results from this very preliminary study support the need for larger, randomized controlled trials with personalized treatment protocols and more careful assessment of adherence to determine the utility of light therapy for postpartum depression and to better characterize possible circadian mechanisms of action. These studies would also help to identify the characteristics of women who are most likely to respond to light therapy during the perinatal period.

\section{Acknowledgments}

Funding: This research was supported by the Gilmore Fund for Sleep Research and Education (University of Michigan) and the National Heart, Lung, and Blood Institute (Swanson; K23HL122461). 


\section{REFERENCES}

Burgess HJ, Wyatt JK, Park M, Fogg LF (2015) Home circadian phase assessments with measures of compliance yield accurate dim light melatonin onsets. Sleep 38:889-897. doi:10.5665/sleep.4734 [PubMed: 25409110]

Corral M, Kuan A, Kostaras D (2000) Bright light therapy's effect on postpartum depression. Am J Psychiatry 157:303-304

Corral M, Wardrop AA, Zhang H, Grewal AK, Patton S (2007) Morning light therapy for postpartum depression. Arch Womens Ment Health 10:221-224. doi:10.1007/s00737-007-0200-1 [PubMed: 17701271]

Gavin NI, Gaynes BN, Lohr KN, Meltzer-Brody S, Gartlehner G, Swinson T (2005) Perinatal depression: a systematic review of prevalence and incidence. Obstet Gynecol 106:1071-1083 [PubMed: 16260528]

LeGates TA, Fernandez DC, Hattar S (2014) Light as a central modulator of circadian rhythms, sleep and affect. Nat Rev Neurosci 15:443-454. doi:10.1038/nrn3743 [PubMed: 24917305]

Lewy AJ et al. (1998) Morning vs evening light treatment of patients with winter depression. Arch Gen Psychiatry 55:890-896 [PubMed: 9783559]

O'Mahen H, Flynn H (2008) Preferences and perceived barriers to treatment for depression during the perinatal period. J Womens Health 17:1301-1309

Okun ML (2016) Disturbed sleep and postpartum depression. Curr Psychiatry Rep 18:66. doi:10.1007/ s11920-016-0705-2 [PubMed: 27222140]

Parry B et al. (2008) Plasma melatonin circadian rhythm disturbances during pregnancy and postpartum in depressed women and women with personal or family histories of depression. Am $\mathbf{J}$ Psychiatry 165:1551-1558 [PubMed: 18829869]

Sharkey KM, Pearlstein TB, Carskadon MA (2013) Circadian phase shifts and mood across the perinatal period in women with a history of major depressive disorder: A preliminary communication. J Affect Disord 150:1103-1108. doi:10.1016/j.jad.2013.04.046 [PubMed: 23706877]

Sockol LE, Epperson CN, Barber JP (2011) A meta-analysis of treatments for perinatal depression. Clin Psychol Rev 31:839-849. doi:10.1016/j.cpr.2011.03.009 [PubMed: 21545782]

van Maanen A, Meijer AM, van der Heijden KB, Oort FJ (2016) The effects of light therapy on sleep problems: A systematic review and meta-analysis. Sleep Med Rev 29:52-62. doi:10.1016/j.smrv. 2015.08.009 [PubMed: 26606319] 
Table 1.

\section{Demographic Characteristics}

\begin{tabular}{lc}
\hline & $\boldsymbol{n}(\boldsymbol{\%})$ or $\boldsymbol{M} \pm \boldsymbol{S D}$ \\
\hline Age & $32.30 \pm 3.27$ \\
Weeks postpartum & $14.4 \pm 9.6$ \\
Breastfeeding at study entry & $6(60)$ \\
Number of children in the home & $2(1)$ \\
Race & \\
$\quad$ Caucasian & $8(80)$ \\
$\quad$ African American & $2(20)$ \\
Marital Status & \\
$\quad$ Married/partnered & $9(90)$ \\
$\quad$ Never married & $1(10)$ \\
Education $\quad$ Some college & $2(20)$ \\
$\quad$ Bachelor's degree or higher & $8(80)$ \\
Employed outside of home & \\
Full-time & $3(30)$ \\
Part-time & $2(20)$ \\
$\quad 215,000$ & $1(10)$ \\
$15,001-30,000$ & $2(20)$ \\
$45,001-60,000$ & $1(10)$ \\
275,001 & $6(60)$ \\
\hline
\end{tabular}


Table 2.

Pre- and -Post Treatment Measures

\begin{tabular}{llllll}
\hline Measure & $\begin{array}{l}\text { Pre-treatment } \\
\boldsymbol{M}(\boldsymbol{S D})\end{array}$ & $\begin{array}{l}\text { Post-treatment } \\
\boldsymbol{M}(\boldsymbol{S D})\end{array}$ & $\boldsymbol{t}$ & $\boldsymbol{d} \boldsymbol{\text { Hedges' effect size } \boldsymbol { g }}$ \\
\hline SIGH-SAD $^{a}$ & $22.13(3.87)$ & $11.25(6.65)$ & $6.23^{* * * *}$ & 7 & 1.89 \\
EPDS $^{a}$ & $11.75(3.62)$ & $7.25(6.86)$ & 2.87 & 7 & 0.78 \\
Diary TST (h:m) & $6: 38(1: 31)$ & $7: 17(1: 19)$ & 1.38 & 7 & 0.42 \\
Diary SE (\%) & $77.38(10.07)$ & $82.63(12.75)$ & $2.87^{*}$ & 7 & 0.43 \\
Actigraphy TST (h:m) & $6: 56(1: 12)$ & $7: 00(0: 50)$ & 0.19 & 6 & 0.06 \\
Actigraphy SE (\%) & $79.69(6.16)$ & $83.47(4.30)$ & 1.47 & 6 & 0.66 \\
DLMO (clock h:m) & $20: 02(1: 21)$ & $19: 56(1: 14)$ & 0.71 & 7 & \\
PAD (h:m) & $7: 57(0: 55)$ & $8: 22(1: 11)$ & 0.21 & 6 & \\
\hline
\end{tabular}

Note. SIGH-SAD= Hamilton Depression Rating Scale, Seasonal Affective Disorder version; EPDS = Edinburgh Postnatal Depression Scale; TST = total sleep time from sleep diary; $\mathrm{SE}=$ percent sleep efficiency from sleep diary; $\mathrm{DLMO}=$ dim light melatonin onset; $\mathrm{PAD}=$ phase angle difference between DLMO and midpoint of sleep per wrist actigraphy.

${ }^{a}$ Sleep items removed from total score.

* $p \leq .05$,

**** $p \leq .001$. 